\title{
An all-fiber, modular, compact Wind lidar for wind sensing and wake vortex applications
}

\author{
Narasimha S. Prasad ${ }^{1} *$, Russ Sibell ${ }^{2}$, Steve Vetorino ${ }^{2}$, Richard Higgins ${ }^{2}$, and Allen Tracy ${ }^{2}$ \\ ${ }^{1}$ NASA Langley Research Center, 5 N. Dryden St., MS 468, Hampton VA, 23681 \\ ${ }^{2}$ Sibelloptics, Boulder CO 80301
}

\begin{abstract}
This paper discusses an innovative, compact and eyesafe coherent lidar system developed for wind and wake vortex sensing applications. With an innovative all-fiber and modular transceiver architecture, the wind lidar system has reduced size, weight and power requirements, and provides enhanced performance along with operational elegance. This all-fiber architecture is developed around fiber seed laser coupled to uniquely configured fiber amplifier modules. The innovative features of this lidar system, besides its all fiber architecture, include pulsewidth agility and user programmable 3D hemispherical scanner unit. Operating at a wavelength of 1.5457 microns and with a PRF of up to 20 $\mathrm{KHz}$, the lidar transmitter system is designed as a Class 1 system with dimensions of 30 "(W) x 46" $(\mathrm{L})$ x 60 "( $\mathrm{H})$. With an operational range exceeding $10 \mathrm{~km}$, the wind lidar is configured to measure wind velocities of greater than $120 \mathrm{~m} / \mathrm{s}$ with an accuracy of $+/-0.2 \mathrm{~m} / \mathrm{s}$ and allow range resolution of less than $15 \mathrm{~m}$. The dynamical configuration capability of transmitted pulsewidths from $50 \mathrm{~ns}$ to $400 \mathrm{~ns}$ allows high resolution wake vortex measurements. The scanner uses innovative liquid metal slip ring and is built using 3D printer technology with light weight nylon. As such, it provides continuous 360 degree azimuth and 180 degree elevation scan angles with an incremental motion of 0.001 degree. The lidar system is air cooled and requires $110 \mathrm{~V}$ for its operation. This compact and modular lidar system is anticipated to provide mobility, reliability, and ease of field deployment for wind and wake vortex measurements. Currently, this wind lidar is undergoing validation tests under various atmospheric conditions. Preliminary results of these field measurements of wind characteristics that were recently carried out in Colorado are discussed.
\end{abstract}

Keywords: Coherent wind lidar, Wake vortex, 3D hemispherical scanner, fiber seed laser, fiber amplifier

\section{INTRODUCTION}

Fast-time wake vortex models have been evaluated for possible operational and research applications related to air traffic management [1-3]. NASA LaRC's Aircraft Vortex Spacing System (AVOSS ) program significantly contributed to the wake vortex and wake turbulence research [4-6]. AVOSS and various other subsequent programs have depended on data provided by wake vortex lidars, specifically WindTracer ${ }^{\circledR}$ for wake research. The wake vortex lidar technology plays a critical role in validating numerical wake models. It is imperative that the measurements such as circulation strength and propagation behavior provided by a wake vortex lidar have to be accurate (close to a true value) and precise (reproducible and repeatable under similar conditions). Furthermore, the wake vortex lidars have to be operationally elegant with acceptable size weight and power (SWaP) as well as economical for a given environment. Current wake vortex lidar technology can be further improved to satisfy these requirements to a large extent. Validation of wake models and corresponding lidar technology enhancements are interdependent. Advancing wake vortex lidar technology would further enable the development of software based processing, display, and decision making tools for rapidly assessing the generation, evolution, and propagation of wake vortices reliably within a desired confidence interval. These tools would then enable the use of wake vortex lidar technology in air traffic control such as for dynamic spacing of aircrafts, wake surfing, aircraft wing design, and many other applications.

The coherent LIDAR (LIght Detection and Ranging) system uses the back-reflected light from aerosols to calculate the Doppler shifted wind velocities. The ability of coherent systems to produce a continuous, real-time 3D scan of wind velocities via detection of backscatter of atmospheric aerosols in clear-air conditions and at long stand-off distances with relatively low pulse energy gives this technology a clear advantage over other atmospheric monitoring technologies.

narasimha.s.prasad@nasa.gov; phone: 757-864-9403 
Lidar systems have proven their value in the remote measurement of spatially resolved atmospheric wind velocities in a number of applications, including the detection of clear-air turbulence, wind shear, microbursts, and aircraft wake vortices [7-12].

Prior to 2002 lidar systems were found only in university laboratories and research institutions. They were experimental, one-of-a-kind systems that required an advanced degree in laser physics to operate. In 1993 an experimental system was deployed at the proposed site of the Hong Kong International Airport by Coherent Technologies, Inc. (CTI) (now known as Lockheed Martin Coherent Technologies, LMCT). Nearly ten years later the first operational Lidar was installed at this same airport with a second Lidar was added in 2007. These early systems were ideal for detecting wind shear during clear air conditions but they were also very expensive and had limited range and flexibility. WindTracer® lidar was first built in 1998 by CTI and has now been installed at several major airports around the world [13]. Government research labs and meteorological institutes have also acquired these units. Following the first lidar installation at the Hong Kong International Airport in 2002 by CTI, eye-safe coherent lidar has been accepted as the instrument of choice for monitoring airplane take offs and landings under clear air conditions when conventional radars does not work. WindTracer ${ }^{\circledR}$ systems are the only long-range Lidar systems that have been effectively used worldwide for more than a decade to detect hazardous winds and aircraft wakes.

At this time, the most mature, tested, and proven commercial Lidar system in the world is WindTracer® lidar systems. Organizations including NASA LaRC have used the data collected from WindTracer® systems for validation of fast time wake models. However, commercially available WindTracer ${ }^{\circledR}$ or custom built lidar systems have several disadvantages such as, they are (a) large and heavy, (b) subjected to high-maintenance, (c) suffer from reliability issues, and (d) often cost-prohibitive. Operational data displays for lidars have not evolved much from the earliest days of Doppler radar. Interaction with the data is generally poor allowing the user only limited zooming or scaling control. Long range, reduced SWaP lidar systems are required for use in difficult circumstances including glide slope measurements.

A coherent lidar system should have ability to dynamically track wake vortices and turbulence along the glide slope path of an aircraft to a much greater resolution than other meteorological measurement systems. Besides, glide slope measurements, wake vortex core measurements and scanning drifting wake vortices under oblique angles are vital for wake research. NASA LaRC is advancing wake vortex lidar technologies for the detection of aircraft-induced wake vortices and turbulence through IRAD, SBIR, NRA and other funding means. The overall goal is to enable the development of novel transceiver architectures with improvements in: range and sensitivity, overall SWaP, system efficiency, reliability, maintenance, accurate wake processing algorithms, real-time data reduction and display schemes. Enhanced graphical display techniques to display a much greater amount of data but to also make the information conveyed by that data more readily apparent to the user are required. Real-time data displays that convey wind phenomena in an intuitive, three-dimensional fashion are desired. It is envisioned that a fiber-based system will offer a significant reduction in SWAP and as such would expand their utility in several applications including air traffic operations to reduce the influence of hazardous wake encounters and harnessing wind energy in wind farms.

\section{NEXT GENERATION ALL-FIBER WIND LIDAR}

To overcome the disadvantages of current systems to a large extent, an eye-safe, long range, versatile wind lidar with allfiber architecture using commercial-off-the-shelf (COTS) components was developed for wind and wake vortex sensing applications. The lidar system, known as Windimager ${ }^{\mathrm{TM}}$, was developed by Sibell Optics under the NASA SBIR funding [14-15] with emphasis to enhancing range, sensitivity, system efficiency and reliability and simultaneously reducing size, weight, and power (SWaP) and maintenance time. Furthermore, it incorporates a novel 3D hemispherical scanner that is integrated into the lidar system. Its total dimensions are 30 "(W) x 46"(L) x 60"(H), weighs $700 \mathrm{lbs}$ and operates over 100-250 VAC, 50/60 Hz. The key features of this all fiber architecture integrated with 3D hemispherical scanner include: modularity, more reliable Telcordia-tested COTS components, turn-key operation, lower maintenance costs, a predominance of line-replaceable units, and cost effective. Besides ground based operations, this wind lidar technology is anticipated to provide a viable technical path for airborne platforms.

Figure 1 illustrates the wind lidar system architecture with optical and electrical interconnects. It can be physically separated into 4 modules: (1) the Scanner Head assembly, (2) the UPS unit, (3) the Environmental Control unit, and (4) the Computer / DAQ unit. Each sub-assembly is an environmentally sealed unit and the four modules can be assembled 
into the full sensor system in a matter of minutes. The compact modular design allows for easy transport and rapid setup. Some of the key features that separates it from the currently available free-space and fiber LIDAR systems are:

- Rapid fire rate that allows for quick and detailed atmospheric profiling

- Pulsed fiber laser technology yields excellent range and wind velocity accuracy

- Variable pulse width operation that adapts for various modes of wind measurements

- Reduced complexity coupled with high-end components maximizes reliability

- Compact, rugged, modular and lightweight construction allows for easy deployment in a variety of harsh environments

- Fiber optic, $1.55 \mu \mathrm{m}$ architecture is easily upgradeable to higher power and greater range configurations while still remaining eye-safe.

- Real-time data-processing and display update.

- User GUI allows multiple application-based operating modes and scan scenarios.

- Custom 3D wind data display enables easy interpretation of atmospheric conditions

- All conduction or convection thermal control - no liquid cooling

- Significantly less expensive than systems with comparable performance

- High electrical efficiency allows the system to run on battery power (8 - 24 hours depending on usage)

The optical interconnects are the polarization maintaining single mode optical fibers. These optical fibers are used in connecting the optical radiation from (a) the optical pulse forming network (PFN) to the couplers and splitters and the Pulse Amplifier Assembly (PAA), (b) the couplers and splitters to the local oscillator and bit monitors, (c) local oscillator to the heterodyne detector, (d) beam receive port to the heterodyne detector, and (d) PAA to the beam launch port. The novel fiber-amplifier network, beam-combined provides over $400 \mu \mathrm{J}$ of energy per pulse. Its features include: improved system sensitivity, continuously variable pulsewidth, compact packaging in a standard 19-inch rack, Reduced complexity coupled with high-end components to maximize reliability, A unique 3D wind data display to enable easy interpretation of atmospheric conditions, compact, rugged, modular and lightweight construction to allow for easy deployment in a variety of harsh environments.

\section{Scanner Head Assembly}

The Scanner Head Assembly consists of the fiber transmitter system, the telescope, and the hemispherical scanner itself, all housed within the scanner dome.

\section{Fiber Transmitter System}

The engine of a lidar sensor is the laser transmitter system, shown in Figure 2 along with the transmit telescope (both part of the Scanner Head Sub-Assembly). Each major component of the sub-assembly is a fiber-coupled, selfcontained unit that can be easily replaced, without the necessity of re-alignment, if the component should fail. The four primary modules of the fiber-laser system are: a master oscillator (MO), based on a telecommunications standard pigtail fiber, continuous wave diode laser that was designed for long-haul telecommunication applications; a fiber-coupled acousto-optic modulator (AOM) that modulates and frequency shifts the MO signal; a fiber-coupled amplifier module to boost the signal to full power and a balanced photodetector/transimpedance amplifier (TIA) module. The entire unit, including the telescope the drivers for the DFB, the optical switch, the AOM, and the EDFA, resides on two 12-inch by 12-inch plates within the Scanner Head. The system offers user selectable continuously variable pulsewidth feature to improve measurement resolution, if needed. The system operates at a wavelength of $1550 \mathrm{~nm}$, a telecommunications industry standard, providing three advantages:

1. Components are available "off-the-shelf" and are less expensive since they are manufactured in large volume.

2. Components have been designed to be environmentally hardened and have been tested to an MTTF metric, making them highly reliable.

3. The $1550 \mathrm{~nm}$ wavelength is at the edge of the near infra-red range, making it more conducive to being eyesafe. The fiber LIDAR system has been evaluated against both ANSI and IEC standards and is rated as a Class 1 transmitter.

The Fiber Transmitter System is a fiber-based Master Oscillator/Power Amplifier (MOPA) architecture. It consists of COTS components including a seed laser, a CW pre-amplifier, a pulse slicer, and a single mode CW EDFA as the first fiber preamplifier. The first preamplifier is then followed by a custom designed second fiber preamplifier and a 
Large Mode Area (LMA) EDFA. The fiber reels holding optical fibers are printed nylon parts. The low power CW MO output is chopped using an Acousto-Optic Modulator (AOM), resulting in a pulsed optical signal, and amplified to full power by a pulsed fiber amplifier. This approach has several advantages:

(a) The MO is a low power module, resulting in low heat dissipation, allowing it to be conduction-cooled.

(b) Pulsed operation is accomplished by chopping the MO output by the AOM. Though some power is sacrificed by this methodology, it is minimal since we chop at low power. The advantage is that, unlike a Q-switched system, a variable pulse-width is available and can be controlled via software control of the AOM driver. This allows the user the ability to configure the sensor according to the desired application. Shorter pulses result in better range resolution for wake vortex measurements.

(c) Narrow linewidth of the MO is maintained by the fiber amplifier, resulting in greater velocity measurement precision.

(d) The system can run between $3 \mathrm{kHz}-20 \mathrm{kHz}$, allowing for a rapid fire-rate (quicker update rate) for applications at shorter distances, such as wake vortex and turbulence detection and measurement.

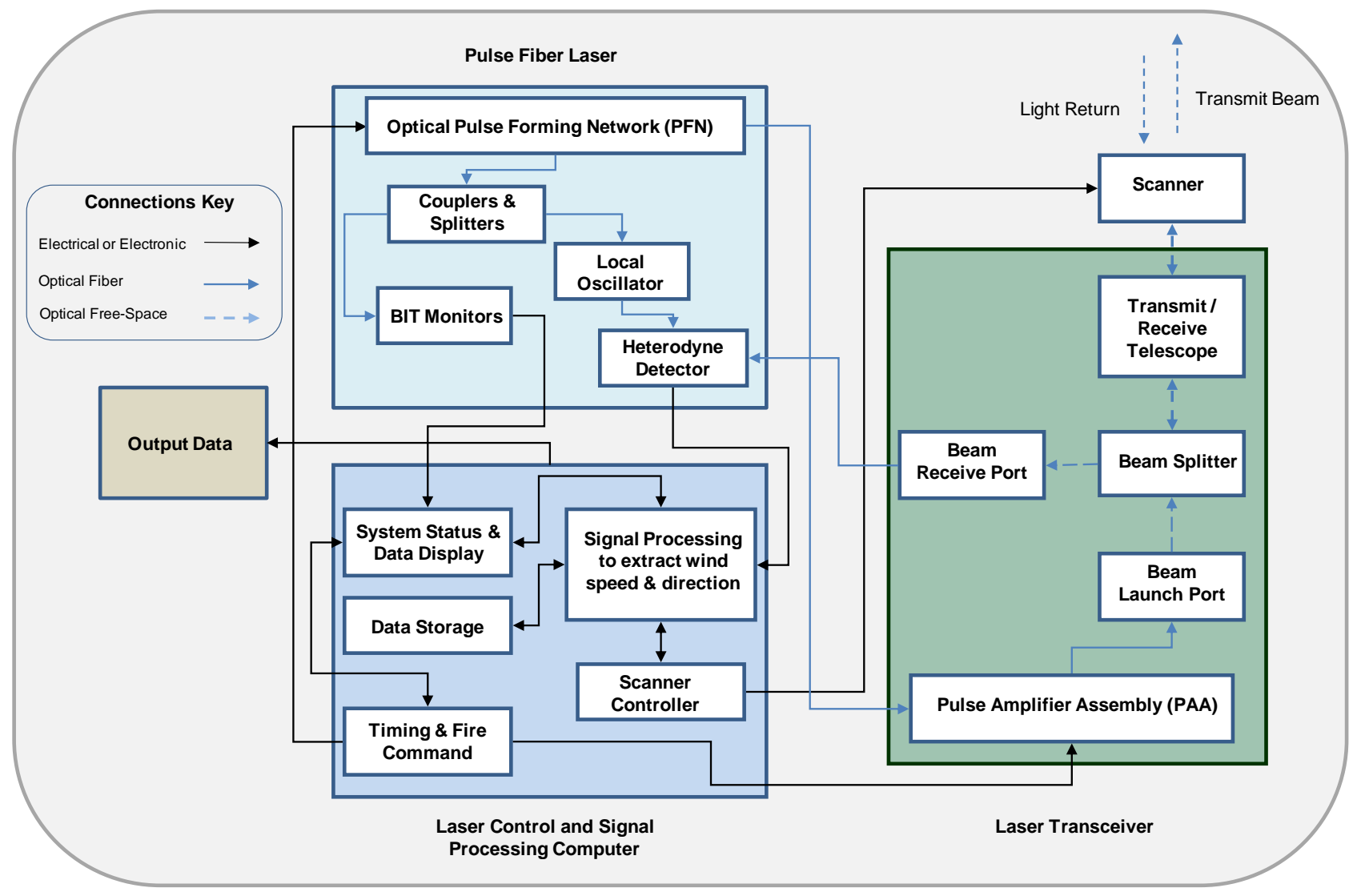

Figure 1. General system architecture of the Windimager ${ }^{\mathrm{TM}}$ lidar system.

The choice of a suitable MO is extremely important as it serves as the seed for the fiber amplifier unit. Two critical MO specifications are the laser linewidth and the Relative Intensity Noise (RIN), both of which affect the radial velocity precision capability of the sensor. The Windimager ${ }^{\mathrm{TM}} \mathrm{MO}$ provides an extremely narrow linewidth $(3 \mathrm{kHz})$ and low RIN $(-145 \mathrm{~dB} / \mathrm{Hz})$.

The fiber-lidar system also incorporates a monitor in which a portion of the signal that has passed through the lidar system, but has not been transmitted, is coupled with a portion of the original local oscillator (LO) signal, allowing for the subtraction of noise and wavefront errors contributed by the lidar system hardware from the received signal, increasing the sensitivity and range of the instrument. 
The Windimager ${ }^{\mathrm{TM}}$, also features two unique components that improve the sensitivity, range and performance of the sensor. The first is the use of a balance detector for both the monitor and the signal detection. Signal detection in any coherent lidar system requires that the LO signal be mixed with the return signal. The beat frequency that results from this coherent mixing contains the Doppler-broadened wind velocity information that is sought. The mixed signal is fed into a photodetector, which converts the optical signal to an electronic signal (current) that is amplified by an integrated pre-amplifier whose voltage output is read and processed.

Typically, alignment is performed by propagating light out of the receiver fiber port and overlapping it with the primary transmit beam. Referred to as the "Back-Propagated Local Oscillator (BPLO)" alignment, this technique requires an overlap path length of 40 meters or greater in order to obtain optimum lidar returns. To increase beam overlap accuracy, two highly sensitivity quad-cell InGaAs detectors capable of resolving a beam's position to within 5 $\mu \mathrm{m}$ was used. By placing the detectors 1 meter apart and using each to monitor the beam positions relative to one another, a 99.7\% overlap was achieved on both detectors. This in turn yielded a pointing error between the beams of 5 $\mu \mathrm{rad}$ - the equivalent to a standard alignment performed over a 100 meter range. In addition to improving the beam overlap accuracy, the detectors and beam steering optics were compact enough to mount on the lidar optical breadboard. This enabled the overlap alignment to be easily checked and optimized whenever and wherever needed. The custom designed detector and its novel use to obtain optimum beam alignment is a significant advancement in the maintainability and manufacturability of lidar systems.

The other unique component of the fiber lidar system is a fiber coupled Optical Switch. The fiber-coupled optical switch is placed between the DFB output and the AOM. The switch will cut off the optical signal to the AOM input port between the primary acoustic pulse generation, thereby preventing a reflected acoustic pulse-generated optical signal.

\section{Telescope Sub-Assembly}

The lidar telescope has two critical functions. The first is to expand the diameter of the input laser beam from a couple of millimeters to several centimeters and to transmit the light in a collimated beam. The second function is to gather scattered laser light from dust and aerosols in the air. In order to maximize the viewing range of the fiber lidar system, the telescope is designed to transmit a collimated beam of light. The large output beam and collection aperture further improve the lidar range. In order to maintain a collimated beam over a wide range of operating temperatures it is critical to keep the distance between the input and output optics of the telescope constant. This is accomplished with a proprietary invar mechanical structure that cancels thermal expansions and contractions. The "reentrant" design ensures that collimation and beam pointing remain fixed regardless of the environment to which the system is subjected.

\section{Hemispherical Scanner}

Unlike all other scanners that use mirrors and rotating wedges to steer beams of light, the Windimager ${ }^{\mathrm{TM}}$ scanner rotates the light source itself - specifically the fiber lidar/telescope assembly. This design has several advantages. Most significantly it reduces the overall complexity of the unit, which in turn improves reliability. In addition, optical scatter and polarization losses are eliminated, thereby improving the lidar performance.

Besides its unique optical design, the scanner is protected by a Lexan® dome and a heated fused silica window, ensuring that it will survive and operate in harsh conditions. The rotation stages have been selected with load capacities several times greater than required. Finally, because of its reduced complexity, the scanner is compact and lightweight. This allows the scanner to be easily mounted on a wide variety of platforms. In addition to data processing and the interactive user interface, the control computer will allow a suite of user selected scanning options as well as the ability to set certain system parameters, depending on the operation. For instance, operating parameters such as range, azimuth, and velocity resolution can be changed in order to see full azimuth, long range scans for wind shear or higher resolution, shorter range glide path scans for wake vortex detection.

The hemispherical scanner is a compact, lightweight, and durable unit that possesses a 0.01 degree repeatable pointing accuracy. By integrating a custom slip ring into the azimuth rotation stage, the scanner is also capable of continuous rotation, which eliminates the need for a complicated and potentially problematic cable take-up mechanism. The slip ring also substantially reduces the wear and tear on the motor itself and when coupled with a structure that has a balanced center of gravity, a mechanical system that has an expected MTBF of 20,000 hours has been achieved. In order 
to prove the full capability of the scanner, it was integrated into an environmental enclosure, which also houses a lidar transceiver and signal processor.

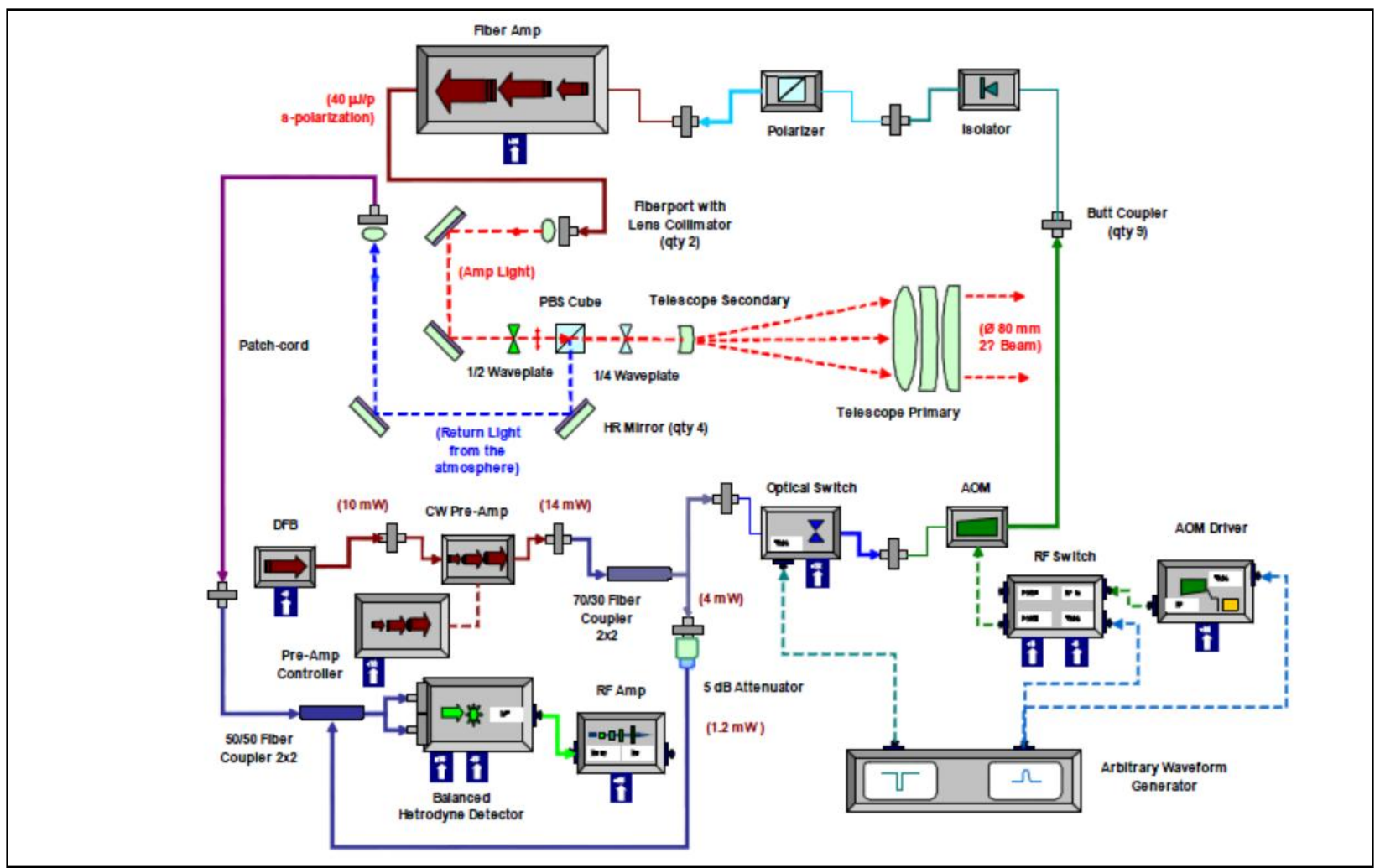

Figure 2. Block diagram of the fiber-coupled transmitter and telescope architecture. The system uses a low energy (50 $\mu \mathrm{J})$ fiber amplifier, includes a pre-amplifier, but does not include a monitor sensor. The modular transmitter components are easily replaced, reducing maintenance and down time.

\section{The environmental Unit}

The environmental control unit is an enclosure temperature control system that is fully insulated and sealed and the pressure operated blower reduces power inrush, using up to 75\% less energy when compared to competitive systems. The control unit is engineered and manufactured to endure the most difficult of environments. It features programmable heating and cooling set points and temperature operating controls. The Windimager ${ }^{\text {TM }}$ is integrated mechanically with the environmental control unit so as to maintain an insulated and sealed lidar system but allow for modularity for greater mobility and ease of maintenance. The unit can be operated remotely by the control computer via an RS-485 cable.

\section{Computer and DAQ system}

A block diagram of the data collection system and display is shown in Figure 3 . The primary input to the data collection and analysis system is the LIDAR balanced heterodyne detector. The detector's output is an analog, radio frequency (RF) signal with amplitude that is proportional to the amplitude of the reflected laser energy. The detector has a bandwidth of $800 \mathrm{MHz}(-3 \mathrm{~dB})$ ensuring that the frequency information present in the reflected laser energy (Doppler shift) will be accurately reproduced in the analog output signal. An interactive 3D data display and control of scan and operating parameters is provided.

Table 1 shows the specifications of the Windimager ${ }^{\mathrm{TM}}$ consisting of transceiver, scanner, and signal processer segments. It also shows the size, weight, environmental conditions and and power requirements. The fully assembled Windimager ${ }^{\mathrm{TM}}$ lidar is shown in Figure 4. 


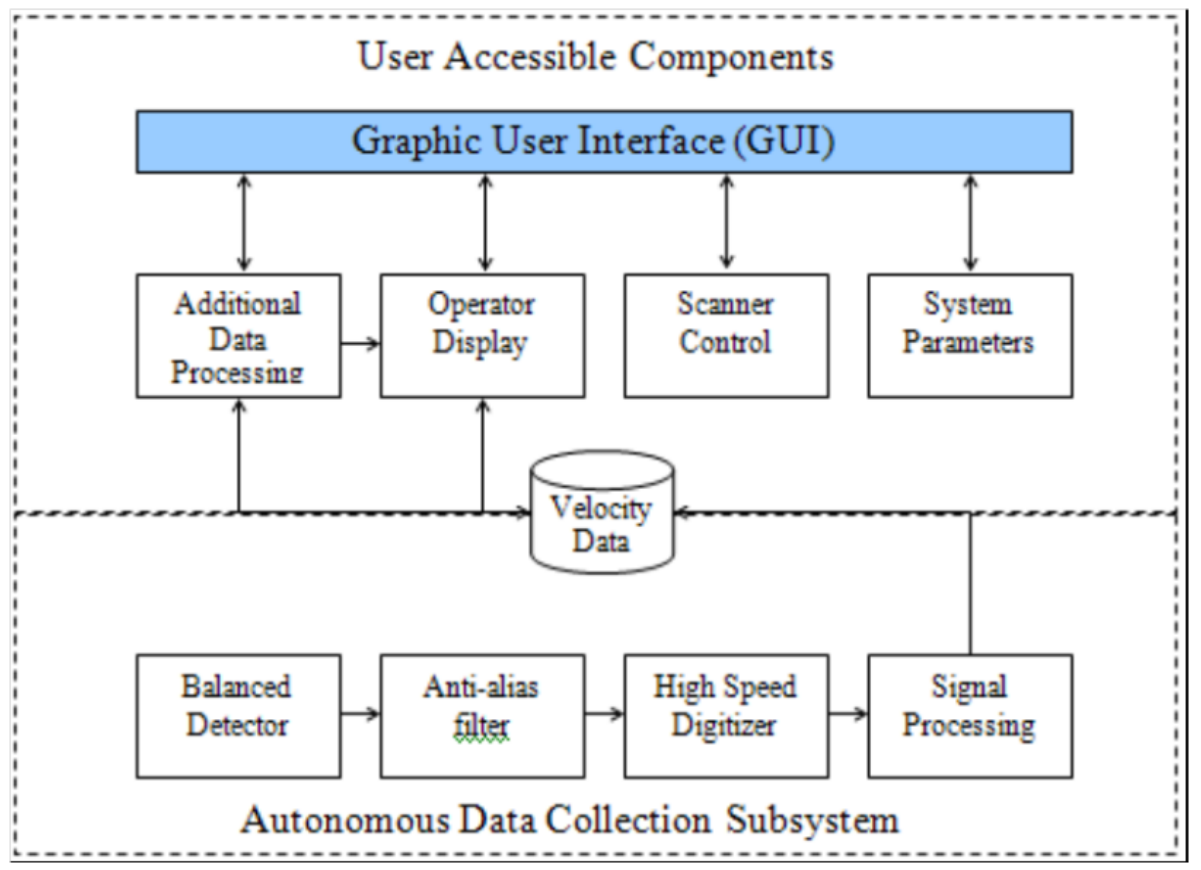

Figure 3. Block diagram of the Computer / DAQ system.

Table 1. Specifications of the all-fiber wind lidar.

\begin{tabular}{|c|c|c|c|}
\hline \multicolumn{4}{|l|}{ Transmitter: } \\
\hline \multirow{2}{*}{\multicolumn{2}{|c|}{$\begin{array}{l}\text { Typical Horizontal Detection Range: } \\
\text { Minimum / Maximum Detection Range: }\end{array}$}} & \multicolumn{2}{|c|}{$400 \mathrm{~m}$ to $10 \mathrm{~km}$} \\
\hline & & \multicolumn{2}{|c|}{$50 \mathrm{~m} / 15 \mathrm{~km}$} \\
\hline \multicolumn{2}{|c|}{ Maximum Radial Velocity: } & \multicolumn{2}{|l|}{$" 127 \mathrm{~m} / \mathrm{s}$} \\
\hline \multicolumn{2}{|c|}{ Velocity Accuracy: } & \multicolumn{2}{|l|}{ " $0.2 \mathrm{~m} / \mathrm{sec}$} \\
\hline \multirow{2}{*}{\multicolumn{2}{|c|}{ Range Resolution: }} & \multicolumn{2}{|c|}{$7.5 \mathrm{~m}$ (minimum), adjustable } \\
\hline \multirow{2}{*}{\multicolumn{2}{|c|}{$\begin{array}{l}\text { Wavelength: } \\
\text { Output Beam Diameter }\left(1 / \mathrm{e}^{2}\right) \text { : }\end{array}$}} & \multicolumn{2}{|c|}{$1547 \mathrm{~nm} " 3 \mathrm{~nm}$} \\
\hline & & \multicolumn{2}{|c|}{$80 \mathrm{~mm}$} \\
\hline \multicolumn{2}{|c|}{$\begin{array}{l}\text { Output Beam Diameter }\left(\begin{array}{ll}1 / \mathrm{e}^{2} & \text { ): } \\
\text { Laser Classification: }\end{array}\right.\end{array}$} & \multicolumn{2}{|l|}{ Class 1} \\
\hline \multicolumn{2}{|c|}{ Pulse Temporal Profile: } & Near Gaus & \\
\hline \multicolumn{4}{|c|}{ Output Pulse Options : } \\
\hline Pulse Width (ns) & Pulse Energy (uJ) & Rep Rate (Hz) & Output Power (mW) \\
\hline 50 & 35 & 20,000 & 700 \\
\hline 90 & 70 & 15,000 & 1050 \\
\hline 170 & 115 & 10,000 & 1150 \\
\hline 200 & 140 & 8,000 & 1120 \\
\hline 330 & 220 & 5,000 & 1100 \\
\hline 400 & 240 & 4,000 & 960 \\
\hline \multicolumn{4}{|c|}{ Scanner } \\
\hline \multicolumn{2}{|c|}{$\begin{array}{l}\text { Scanner } \\
\text { Elevation Scan Range / Rate: }\end{array}$} & \multicolumn{2}{|c|}{$360 f$ continuous, $40 f / \sec (\max )$} \\
\hline \multicolumn{2}{|c|}{ Azimuth Scan Range / Rate: } & \multicolumn{2}{|c|}{$360 f$ continuous, $40 \mathrm{f} / \mathrm{sec}$ (max) } \\
\hline \multicolumn{2}{|c|}{ Unidirectional Repeatability: } & \multicolumn{2}{|c|}{0.002 degrees } \\
\hline \multicolumn{2}{|c|}{ Min Incremental Motion: } & \multicolumn{2}{|c|}{0.001 degrees } \\
\hline \multicolumn{2}{|c|}{ Absolute Positional Accuracy: } & \multicolumn{2}{|c|}{ " 0.01 degrees } \\
\hline \multicolumn{2}{|l|}{ Scan Resolution: } & \multicolumn{2}{|c|}{ Up to 10,000 points / PPI scan } \\
\hline \multicolumn{2}{|l|}{$\begin{array}{l}\text { Scan Modes: } \\
\text { combination }\end{array}$} & complex group m & tion, or \\
\hline \multicolumn{2}{|l|}{ Scan Patterns } & Irammable & \\
\hline Rotation Stages MT & & 20,000 hou & \\
\hline Optical Clear Apert & & $101 \mathrm{~mm}$ & \\
\hline
\end{tabular}

Signal Processor:

Operating System:

Graphical User Interface:

System

CPU:

Memory:

Hard Disk Drive:

Digitizer Card:

Timing:

Additional Sensors:

Input Devices:

Network:

Size \& Weight:

System Dimensions:

System Weight:

Environmental:

Operating Temperature: $\quad-40^{\circ}$ to $+55^{\circ} \mathrm{C}$

Storage Temperature: $\quad-25^{\circ}$ to $+40^{\circ} \mathrm{C}$

Cooling System: Air-cooled

Power Requirements:

Input Power:

Service Circuit Req:

Voltage (single phase):
MS Windows

NOTUS Data Collection

Intel Core i7-820QM, $1.73 \mathrm{GHz}$ 4 GB DDR3

Up to 24 TB, RAID array $750 \mathrm{MS} / \mathrm{s}$ sample rate, $16 \mathrm{MB}$

RAM, 8 bits/sample

GPS, IEEE-1588 or IRIG-B

GPS, Compass (Optional)

Ethernet

100BaseT

30-in $(\mathrm{W}) \times 46$-in $(\mathrm{L}) \times 60$-in $(\mathrm{H}$ $800 \mathrm{lbs}(364 \mathrm{~kg})$

1100 W Max, 550 W Typical

20 Amps

$100-250$ VAC, $50 / 60 \mathrm{~Hz}$ 

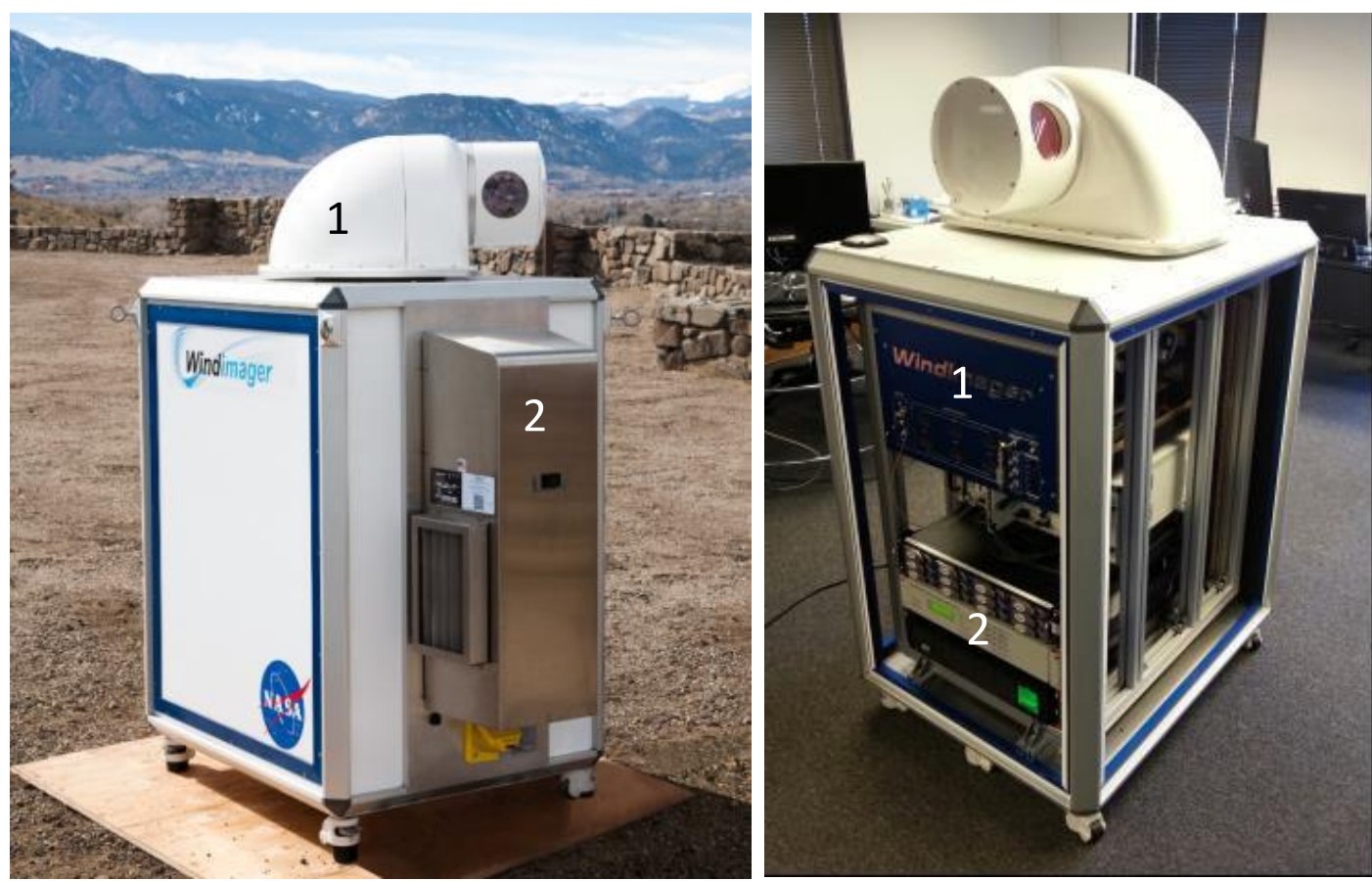

Figure 4. The Windimager ${ }^{\mathrm{TM}}$ lidar unit. Left Picture: (1).Hemispherical scanner, (2). Air cooled unit. Right Picture The internal arrangement. (1).The idar transceiver chassis. (2). The UPS unit

from $50 \mathrm{~ns}$ to $400 \mathrm{~ns}$ and the user friendly GUI with programmable scan pattern (0-180 Elevation and $360^{\circ}$ azimuth) allows the operator to limit the operational range as desired. The UPS battery pack is capable of providing up to 10 hours of operational power to the system. The lidar system with fiber amplifier modules has no alignment issues and has only one switch to turn on. In the following section, the preliminary results of field tests carried out near Boulder, $\mathrm{CO}$ are discussed.

\section{FIELD TEST RESULTS}

Figure 5 shows the heat map (Range, $\mathrm{km}$ vs. Wind speed, $\mathrm{m} / \mathrm{s}$ ) representation of a typical horizontal single line-ofsight (LOS) returns collected by the Windimager ${ }^{\mathrm{TM}}$ lidar located facing the Rocky mountain range near Boulder, CO. From this heat map, the hard target representing Rocky mountains at a distance greater than $9 \mathrm{~km}$, winds up to $8.5 \mathrm{~km}$, and a wind shear event around $1.5 \mathrm{~km}$ can be seen. Figure 6 shows four different scenarios of single LOS returns along with estimated aerosol density values obtained from nearby pollution monitoring center. For results shown in Figures 5 and 6, the pulsewidth was $400 \mathrm{~ns}$, pulse energy was $240 \mu \mathrm{J}$, PRF of $4 \mathrm{kHz}$, and data averaging of 2000 shots $(0.25$ seconds). In these four pictures, hard target returns and wind events were consistently captured. Also shown in these heat maps are the particulate counts that were obtained from the pollution monitoring station set up by the state of Colorado located near Ft. Collins for measuring air quality. The station provides hourly data of aerosol particulate counts. An attempt was made to correlate the lidar visibility and measured aerosol particulate counts, so that one could relate the two as a way of predicting the lidar performance based on existing measurements. However, the closest measuring site to the lidar location was just over 19 miles away and a good correlation was seen when there was a very large scale weather event such as a strong cold front which affects the entire area. Smaller events, such as snow squalls and thunderstorms, seem to have a much more local effect and show almost no correlation between the two measurements. The other issue is one of reliability due to unavailability of data points from the station at certain times. As such, it is virtually impossible to correlate these two data sets due to the separation distance and inconsistency of the data. 


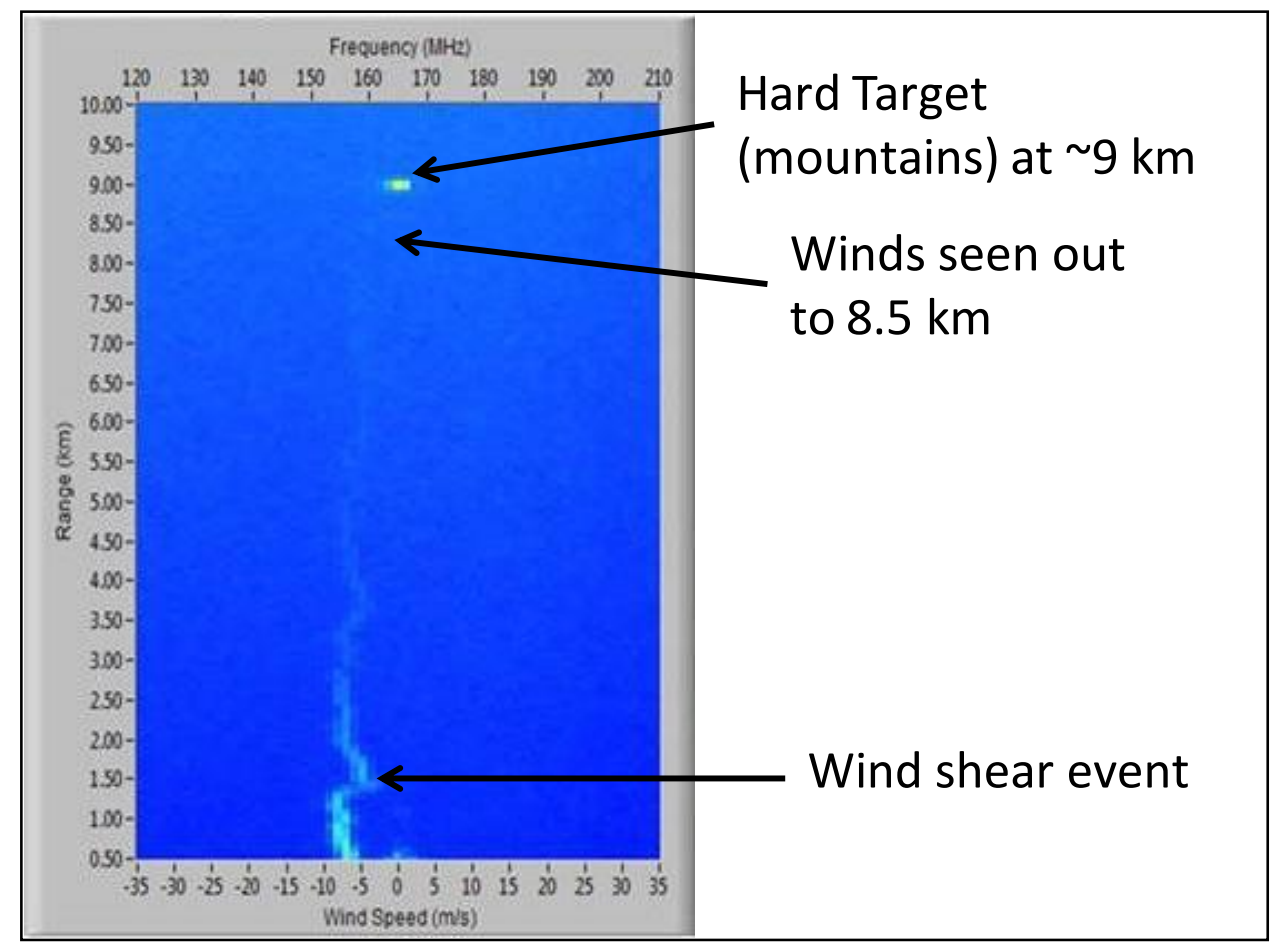

Figure 5. Horizontal single line-of-sight (LOS) returns collected by the Windimager $^{\mathrm{TM}}$ lidar near Boulder, CO.

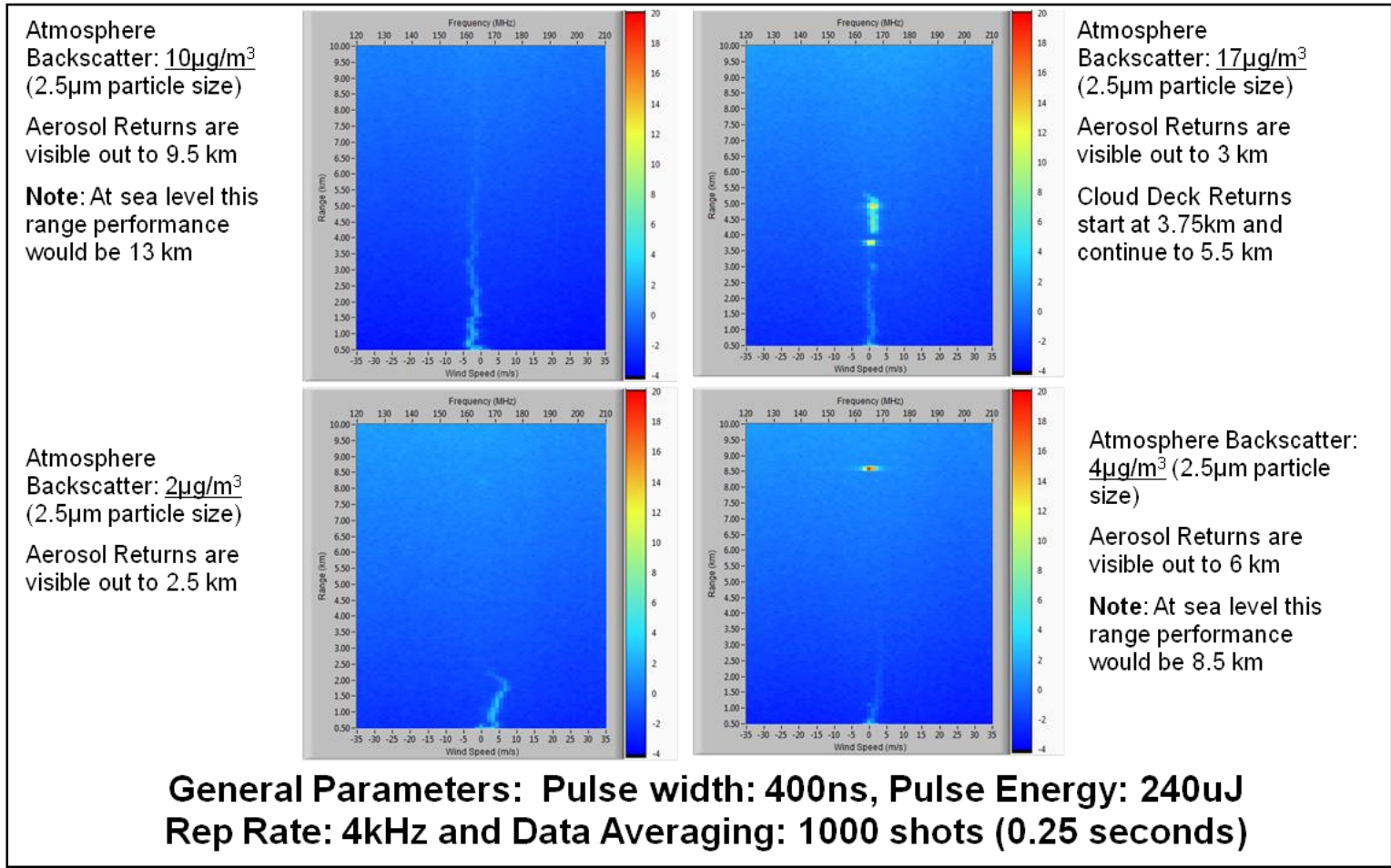

Figure 6. (a) Top Left: Horizont single LOS aerosol returns. (b) Top right: Vertical single LOS aerosol returns, (c) Bottom left: Horizontal single LOS aerosol returns during rain storm, and (d) Bottom right: Horizontal single LOS aerosol returns after rain storm.

The lidar system provides Plan Position Indicator (PPI), Range Height Indicator (RHI) data displays, and sector scans. Figure 7 shows typical PPI scans that were routinely obtained and analyzed. Figure 8 shows a 30 degree sector scan. 
The scan was taken with the elevation angle set at 2 degrees above the horizon, pulse averaging set to 2000 shots, the pulse energy set to $280 \mu \mathrm{J}$, the pulsewidth set to $400 \mathrm{~ns}$, and the PRF set to $4 \mathrm{kHz}$. In this image, broken aerosol returns out to $5 \mathrm{~km}$ and hard target mountain returns starting at $10 \mathrm{~km}$ are seen. Field tests are continuing to establish the system validity for wind measurements and subsequently for wake measurements.

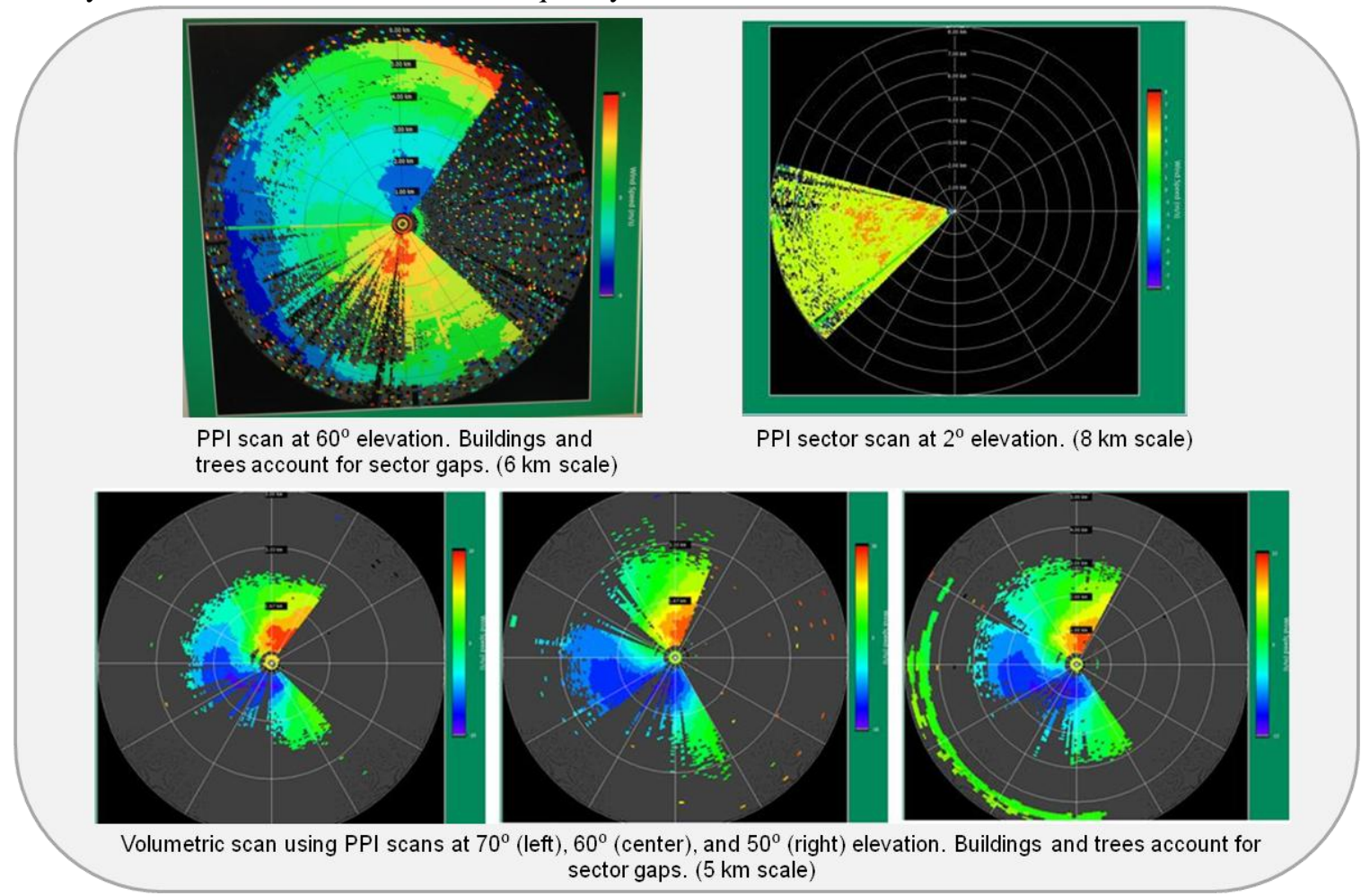

Figure 8. Typical scans offered by the wind lidar.

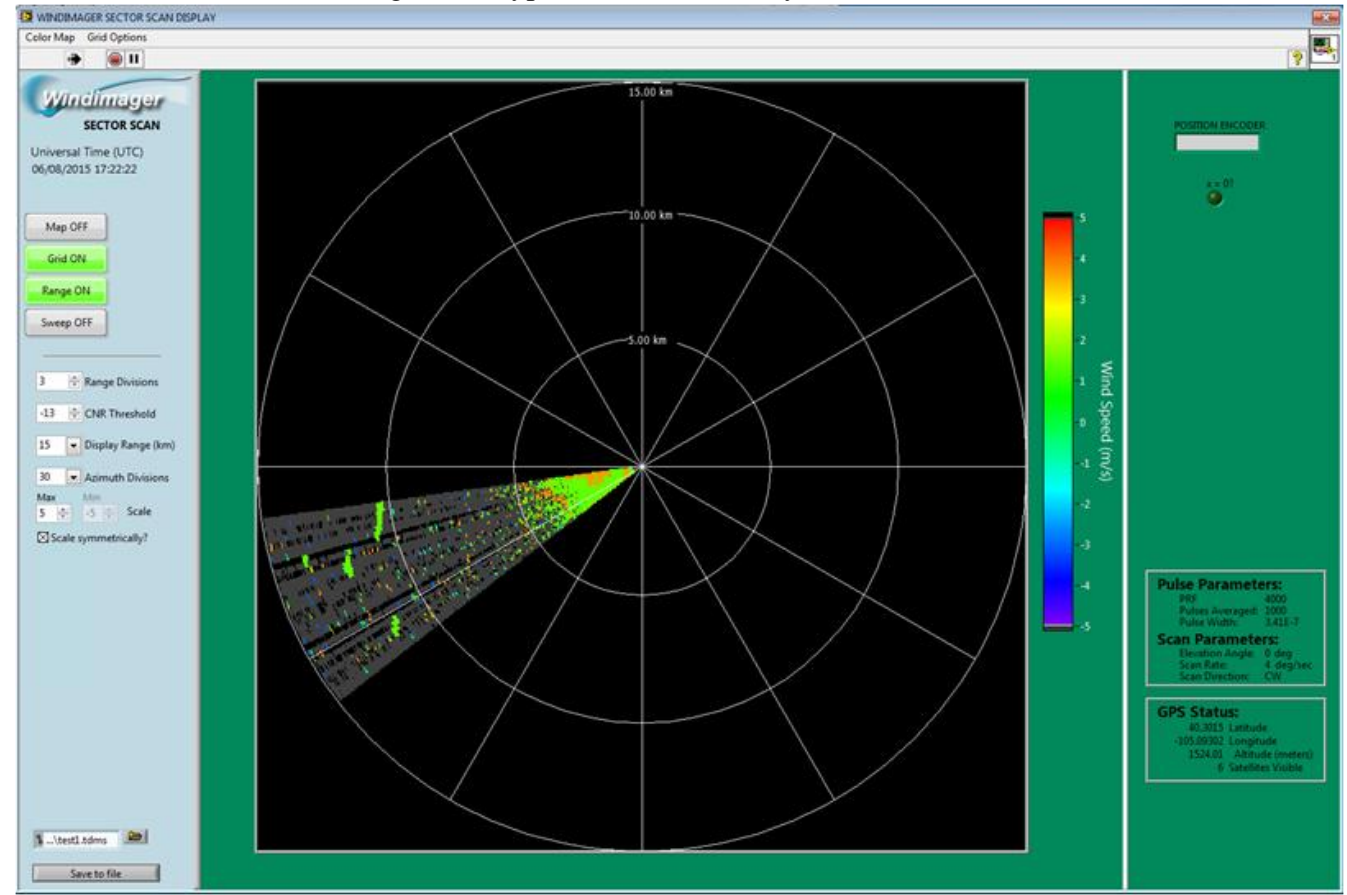

Figure 9. The 30 degree sector scan with $5 \mathrm{~km}$ scale showing aerosol returns. Circles are drawn every $5 \mathrm{~km}$. The sector scan was taken with the elevation angle set at 2 degrees above the horizon. 
The compact all fiber, Windimager ${ }^{\mathrm{TM}}$ class of technology system has the potential to provide a developmental path for several applications. They include (a) Wind hazard monitoring for clear-air wind, shear, and gust fronts imaging in fine detail, (b) Wake vortex monitoring for air safety and with dynamic pulsewidth capability, high fidelity direct measurement of wake vortex velocity distributions, (c) chem-bio tracking by monitoring the propagation of hazardous aerosols, (d) hard target detection with applications in navigational hazard avoidance specifically in radar quiet zones, (e) ballistic winds for enhanced targeting using compensated high-resolution wind data and (f) cloud base height and density measurements.

\section{SUMMARY AND CONCLUSIONS}

The salient features of the development of an all-fiber, modular, compact architecture based wind lidar with an integrated, novel 3D hemispherical scanner for wind sensing and wake vortex applications is presented. Based on the high repetition rate 1.55 micron fiber laser transmitter system consisting of seed laser and fiber amplifier modules, the wind lidar is configured to measure wind velocities greater than $120 \mathrm{~m} / \mathrm{s}$ with a resolution of $0.2 \mathrm{~m} / \mathrm{s}$ over ranges great than $10 \mathrm{~km}$. Pulsewidth agility has been incorporated to provide high spatial resolution measurements. The scanner provides 360 degrees of azimuth and 0-180 degree elevation angles at a rate of 40 degree per second and a positional accuracy of 0.01 degrees. The lidar system is air cooled with overall dimensions of 30" $(\mathrm{W})$ x 46" (L) x 60"(H). Field test results carried out under various meteorological conditions in Boulder, $\mathrm{CO}$ area are presented. Techniques to further reduce background detector noise are being implemented. Validation studies for establishing system performance are progressing. Wake algorithms are being developed. Developmental plans for airborne and shipboard implementation are underway.

\section{REFERENCES}

[1] Proctor, F.H., "Evaluation of Fast-Time Wake Vortex Prediction Models," January 2009, AIAA 2009-0344.

[2] Proctor, F.H., and Switzer, G.F., "Numerical Simulation of Aircraft Trailing Vortices," 9th Conf. on Aviation, Range and Aerospace Meteorology, American Meteorology Society, September 2000, pp. 511-516.

[3] Proctor, F. H., "The NASA-Langley Wake Vortex Modeling Effort in Support of an Operational Aircraft Spacing System," January 1998, AIAA 98-0589.

[4] Hinton, D.A., "Aircraft Vortex Spacing System (AVOSS) Conceptual Design," August 1995, NASA TM-110184.

[5] Perry, R.B., Hinton, D.A., and Stuever, R.A., "NASA Wake Vortex Research for Aircraft Spacing," 35th Aerospace Sciences Meeting \& Exhibit, January 1997, AIAA 97-0057.

[6] Hannon, S. M., M. W. Phillips, J. A Thompson, and S. W Henderson, "Pulsed Coherent Lidar Wake Vortex detection, Tracking and Strength Estimate in Support of AVOSS," NASA First Wake Vortex Dynamic Spacing Workshop - NASA CP-97-206235, 1997.

[7] Huffaker, R.M. and R.M. Hardesty, "Remote sensing of atmospheric wind velocities using solid-state and CO2 coherent laser systems", Proc. of the IEEE, vol 84, No. 2, 181-204, 1996.

[8] Killinger, D.K. an N. Menyuk, "Laser remote sensing of the atmosphere", Science 235, 37, 1987.

[9] Technical Assistance Document for the NATTS Program, Revision 2, April 1, 2009, p. 136.

[10] R. Targ, M. J. Kavaya, R. M. Huffaker, and R. L. Bowles, "Coherent LIDAR airborne windshear sensor: performance evaluation," Appl. Opt. 30, 2013 (1991).

[11] Srivastava V., et. Al. "Wavelength dependence of backscatter by use of aerosol microphysics and LIDAR data sets: application to 2.1-um wavelength for space-based and airborne LIDARs," Appl. Opt. 40, No. 27, 4759-4769 (2001).

[12] Van Trees, Detection, Estimation, and Modulation Theory, Part. I, Wiley and Sons (1968) (See Eq. 2.516).

[13] Sammy W. Henderson and Stephen M. Hannon , "Advanced coherent lidar system for wind measurements," Proc. SPIE 5887, Lidar Remote Sensing for Environmental Monitoring VI, 58870I (16 September 2005).

[14] "Next Generation Fiber Coherent Lidar System for Wake Vortex Detection" Contract \# NNX12CA74C, The NASA SBIR Phase II final report, Sibell Optics, 2014.

[15] "Novel Hemisphere Scanner for a Coherent Fiber LIDAR System", Contract \# NNX12CA73C, The NASA SBIR Phase II final report, Sibell Optics, 2014. 